\title{
Le laboratoire au cabinet médical: état des lieux trois ans après l'Ordonnance de l'OFSP
}

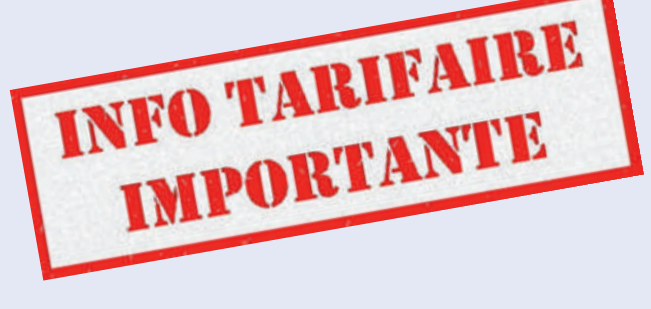

\author{
L'Ordonnance du Conseil fédéral décrétée il y a trois ans par l'Office fédéral de \\ la santé publique (OFSP) concernant la liste des analyses est en vigueur depuis le

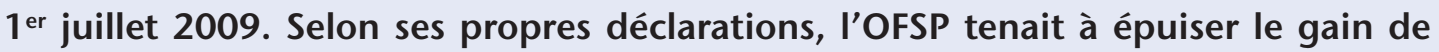 \\ 100 millions rendu possible grâce à l'efficacité des progrès technologiques. Fort de \\ cet argument, il a tarifé toute la liste des analyses d'après les exigences et l'infra- \\ structure des laboratoires mandatés.
}

Anton Prantl ${ }^{a}$, Ernst Gähler ${ }^{b}$

a Président de direction Caisse des médecins

b Dr méd., vice-président de la FMH, responsable du domaine Tarifs ambulatoires et conventions Suisse
Correspondance:

$\mathrm{FMH}$

Froburgstrasse 15

CH-4600 Olten

Tél. 0313591230

Fax 0313591238

tarife.ambulant[at]fmh.ch

\section{Evolution du laboratoire au cabinet médical durant les trois années écoulées}

L'OFSP publie sur son site internet les points les plus importants relatifs à l'évolution du laboratoire au cabinet médical depuis l'entrée en vigueur de l'Ordonnance:

La nouvelle liste des analyses est restée sans effet chez les laboratoires mandatés et, depuis son introduction, leur volume a augmenté chaque année de plus de $5 \%$ et même de $8,2 \%$ durant le premier semestre 2012 par rapport à 2011.

- Depuis l'introduction de la liste des analyses, les médecins en libre pratique ont vu leurs chiffres d'affaires baisser de $20 \%$. Le volume n'a augmenté que de $2 \%$ environ par année; le premier semestre 2012 enregistre également une augmentation plutôt modérée $(2,4 \%)$ par rapport à 2011.

- Dans le secteur ambulatoire des hôpitaux également, des sommes importantes sont facturées de plus en plus pour des analyses de laboratoire.
Nous ne disposons malheureusement d'aucune donnée précise.

Cette situation est absolument symptomatique. Par ses mesures, l'OFSP qui se reconnaît partie prenante des soins de base empêche tout d'abord une gestion appropriée et économiquement adéquate du laboratoire au cabinet médical et remet ensuite le gain d'efficacité à ceux qui peuvent effectivement le réaliser en raison des économies d'échelle. De surcroît, il passe sous silence la situation des hôpitaux en ce qui concerne la saisie des coûts de laboratoire et ceux des médicaments de leur secteur ambulatoire.

\section{Situation des médecins en libre pratique}

Il ne sert à rien de continuer à se plaindre du travail inadéquat et des connaissances professionnelles lacunaires qui caractérisent la liste des analyses, tout cela a été confirmé et prouvé par le monitorage effectué pendant plus de deux ans. Contrairement au point de vue de santésuisse et de l'OFSP, la FMH n'a cessé de

\section{Figure 1}

Nombre d'analyses au cabinet médical. Le graphique 1 montre - avec suffisamment de distance par rapport à l'intervention de I'OFSP - l'évolution du nombre d'analyses effectuées par mois au laboratoire du cabinet médical et le nombre de cabinets qui réalisent encore des analyses. Aucun changement significatif n'est à constater. Ce graphique confirme que la façon de travailler des médecins n'a pas changé malgré les coupes intervenues. Les laboratoires existants sont maintenus. Les jeunes médecins qui débutent dans l'activité ambulatoire d'un cabinet vont certainement se demander, dans ces circonstances, $s^{\prime}$ ils tiennent vraiment à gérer un laboratoire qui ne couvre pas les frais. Mais il faudra attendre un certain temps avant d'en apercevoir les conséquences.

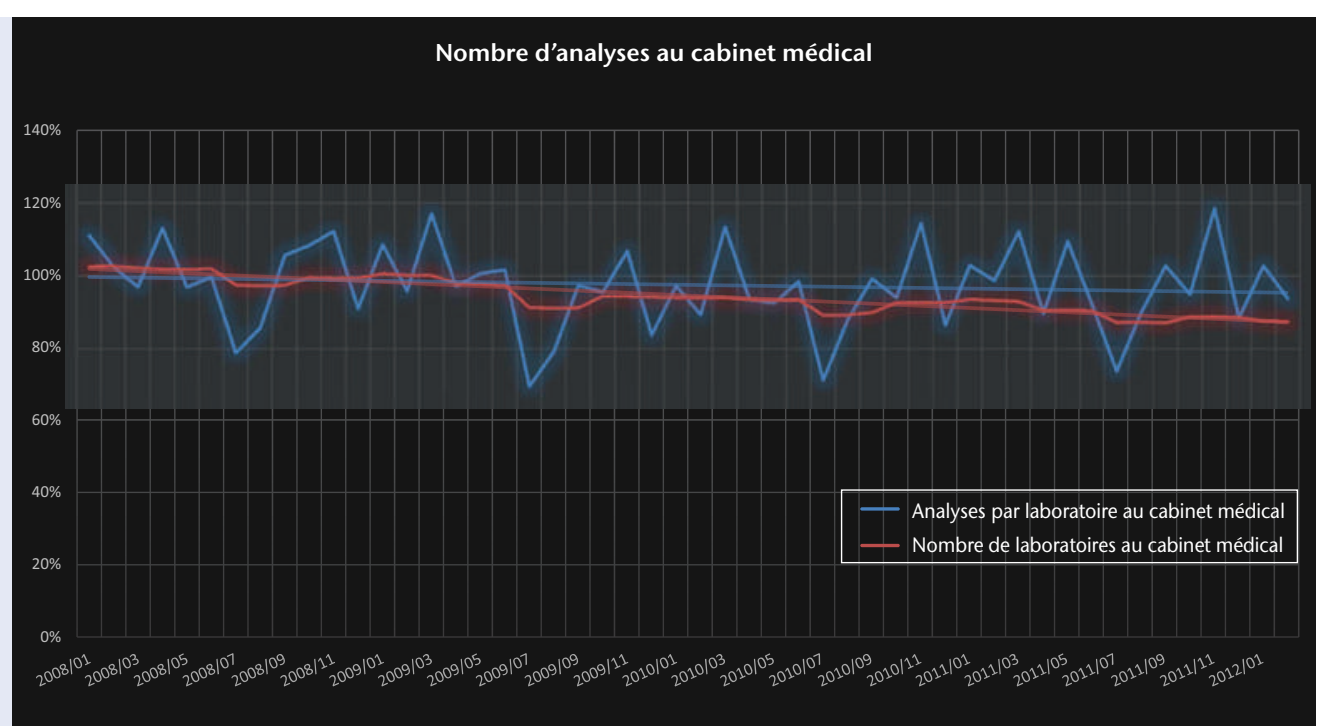




\section{Figure 2}

Evolution indexée du chiffre d'affaires du laboratoire au cabinet médical.

Le graphique 2 montre - avec suffisamment de distance par rapport à l'intervention de l'OFSP - l'évolution indexée de l'ensemble des chiffres d'affaires, du chiffre d'affaires par laboratoire au cabinet médical et du chiffre d'affaires par analyse. On constate très clairement la nette baisse de $20 \%$ au moment de l'introduction de la nouvelle liste et l'absence - incompréhensible du point de vue économique - de toute compensation ultérieure par les médecins. Ce graphique montre ainsi de façon admirable que les médecins placent au premier rang le bien-être des patients et la qualité élevée de leur travail.
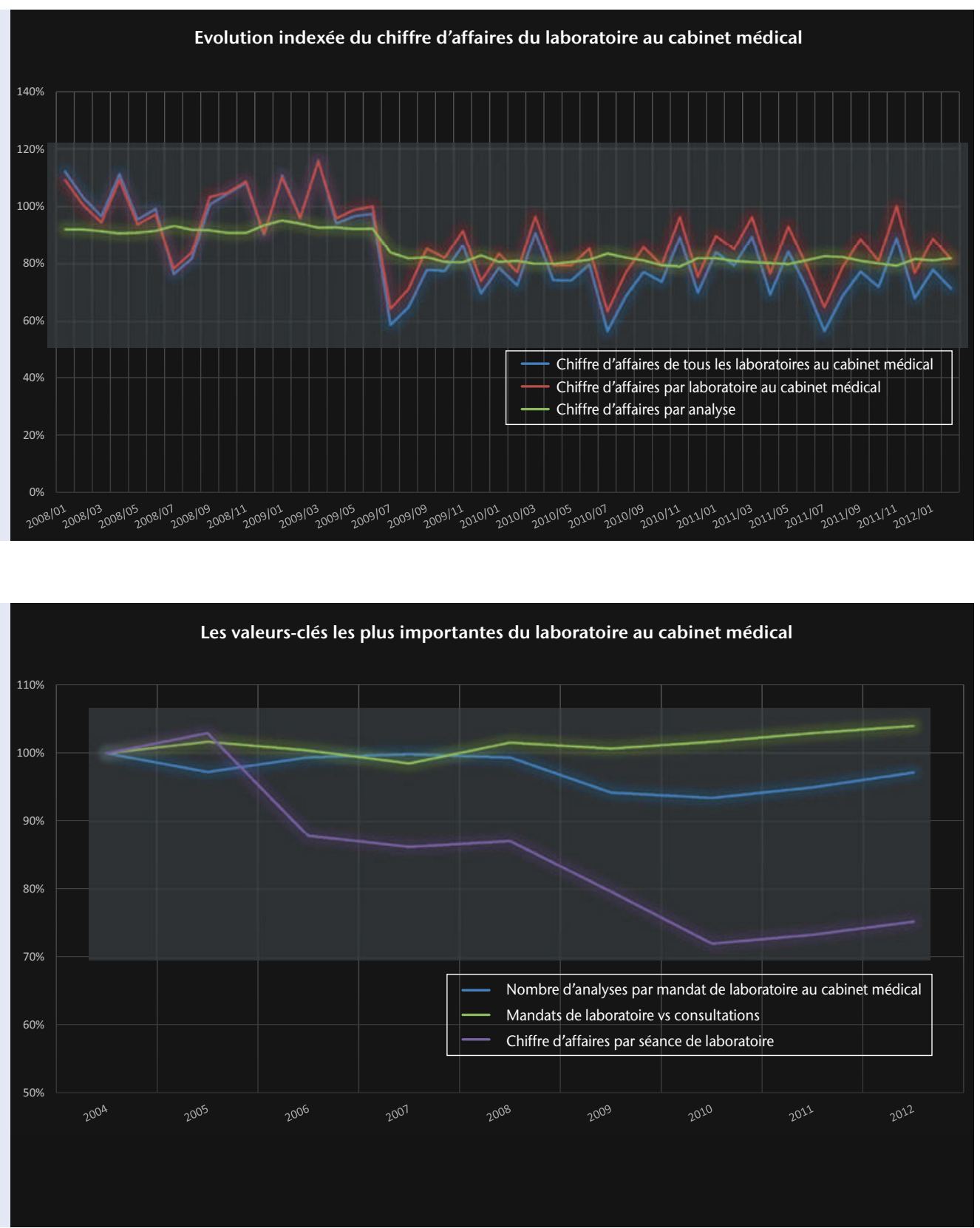

\section{Figure 3}

Les valeurs-clés les plus importantes du laboratoire au cabinet médical.

Le graphique 3 montre - avec suffisamment de distance par rapport à l'intervention de I'OFSP - l'évolution indexée du nombre d'analyses par mandat de laboratoire au cabinet médical, les mandats de laboratoire par consultation et le chiffre d'affaires par séance de laboratoire. Si les médecins étaient sensibles aux incitations économiques, ils devraient réagir à ces pertes - en ordonnant davantage d'analyses - Or le nombre de mandats de laboratoire par rapport au nombre de consultations est resté presque constant

- en ordonnant davantage d'analyses par mandat de laboratoire - Or le nombre des analyses par mandat se rapproche à nouveau de la valeur à long terme

- en ordonnant davantage d'analyses plus chères - Or le chiffre d'affaires par séance de laboratoire a chuté de 30\% et ne remonte guère. rappeler que le laboratoire au cabinet médical n'est pas une «source de revenus» supplémentaire mais qu'il est une nécessité médicale et sert à poser des diagnostics, à prescrire une thérapie appropriée et à la contrôler. Le laboratoire de la médecine libérale est un instrument permettant d'assurer la qualité des soins ambulatoires. De ce fait, le comportement des médecins n'a pas fondamentalement changé dans leur laboratoire malgré la situation critique actuelle et la chute massive des prix. Nous l'avons vérifié avec précision dans les graphiques ci-avant.

\section{Conclusion}

L'intervention de l'OFSP par voie d'ordonnance a provoqué une évolution erronée involontaire, d'ailleurs prédite par le corps médical qui ne lui accorde pas de satisfecit. Nos estimations sont catastro- phiques pour les médecins libres praticiens et elles nous incitent à exiger expressément et avec force que l'OFSP corrige rapidement cette situation intenable.

Il est inacceptable que l'OFSP abuse à ce point de sa compétence pour fixer les prix de manière aussi unilatérale et sans utilité médicale pour le patient aux fins d'économies supposées. En première ligne, c'est la qualité des soins médicaux ambulatoires qui est menacée par de telles mesures. Nous attendons de l'OFSP qu'il retranscrive et rémunère dans la liste des analyses le laboratoire au cabinet médical de façon correcte et avec fair-play. Cette situation insatisfaisante dans le domaine de la liste des analyses est en contradiction flagrante avec l'intention constamment réitérée de l'OFSP d'améliorer et de renforcer la situation des médecins de famille et ceux de premier recours par des mesures appropriées et ciblées. 